\title{
Homology Curve Complex
}

\author{
Ningthoujam Jiban Singh ${ }^{{ }^{*}}$, Himadri Kumar Mukerjee ${ }^{2}$ \\ ${ }^{1}$ Department of Mathematics, Assam University, Silchar, India \\ ${ }^{2}$ Department of Mathematics, North-Eastern Hill University, NEHU Campus, Shillong, India \\ Email: \{njiban, himadrinehu\}@gmail.com
}

Received October 22, 2011; revised November 1, 2011; accepted November 12, 2011

\begin{abstract}
A homological analogue of curve complex of a closed connected orientable surface is developed and studied. The distance in this complex is shown to be quite computable and an algorithm given (Theorem 3.5). As an application of this complex it is shown that for a closed orientable 3-manifold, and any of its Heegaard splittings, one can give an algorithm to decide whether the manifold contains a 2-sided, non-separating, closed incompressible surface (Theorem 1.1).
\end{abstract}

Keywords: Homology Curve Complex; Heegaard Splittings; Incompressible Surface

\section{Introduction}

Jaco and Oertel ([1]) gave an algorithm to decide if a 3-manifold is a Haken manifold. Their algorithm is an offshoot of an algorithm given by Haken himself. Haken has developed in 1960's ([2]) an algorithm to decide if a fundamental normal surface of a given presentation of a compact irreducible 3-manifold is injective. Any normal surface can be constructed from these fundamental normal surfaces and if the input is a normal surface then by successive application of the above algorithm one can check if the normal surface is injective. But one does not know for how many normal surfaces (finite or infinite) this algorithm has to be run. Jaco and Oertel ([1]) produced a finite collection of constructible normal surfaces (test surfaces) only on which this algorithm has to be run. Results of similar flavour has been found by Jaco, Letscher, and Rubinstein [3] and many others.

We, on the other hand, give here an algorithm whose input is any Heegaard splitting of the given manifold. We develop a homological version of curve complex, which we call "homology curve complex".

The term homology curve complex used in this paper is inspired by the term used in the set of open problems posed by Joan Birman in the arXiv preprint [4], page no. 8 , line 12 , but is different from what she meant there. This term has also been used recently by Ingrid Irmer in his arXiv preprint [5] with a different meaning.

We prove that for surfaces of genus greater than one, this complex is connected (3.3), hence a notion of distance between vertices, which we call "H-distance" can be defined. We give an algorithm which returns H-dis-

${ }^{*}$ The first author acknowledges CSIR, India, for financial supports under grant F. No. 9/347(159)/2k3-EMRI dated 13.09.2003. tance of two given vertices, theorem (3.5). Using this complex we define an analogue of the Hempel distance of a Heegaard splitting of a 3-manifold. This distance is then used to decide if the manifold contains a closed, two-sided incompressible surface or not, a homological analogue of Johnson and Patel ([6], lemma 4), which also extends to genus 2 in our case. Finally an algorithm is given which takes as its input a Heegaard splitting of a given closed connected orientable 3-manifold and decides if this distance is zero or not in polynomial time.

Every closed connected orientable 3-dimensional manifold $\mathrm{M}$ admits a Heegaard splitting, i.e., a decomposition into two orientable handlebodies $V$ and $V^{\prime}$ which meet along their boundary. This common boundary is called a Heegaard surface. Alternately, we may view $\mathrm{M}$ as obtained from the two handlebodies by identifying their boundaries under a homeomorphism $f: \partial V \rightarrow \partial V^{\prime}$. Heegaard splittings are a convenient way to define a 3manifold, but a priori it is difficult to get structural information about the manifold from them. It is in this light the following result assumes importance.

\section{Theorem}

(Theorem 4.1) Given a closed, connected, orientable 3manifold $M$ and a Heegaard splitting $M=V \cup_{\Sigma g} V^{\prime}$ of genus $g>1$, there is an algorithm, which runs in polynomial time, to decide whether $M$ contains a nonseparating, 2-sided, closed incompressible surface.

In Section 2 we give a brief recollection of complex of curves and some related concepts. We introduce in Section 3 an analogue of curve complex, "homology curve complex", associated with the surface $\sum$ and a notion of distance, "H-distance", and prove several results de- 
scribing their properties including a result giving an algorithm to compute the $\mathrm{H}$-distance between a pair of vertices: see lemma (3.2), lemma (3.3), theorem (3.5), proposition (3.6), proposition (3.7). Section 4 starts with the introduction of the notion of an analogue of the Hempel distance of a Heegaard splitting followed by a recollection of complete meridian systems and some results related to these. Finally, we complete the proof of theorem (1.1) giving an algorithm which takes as its input a Heegaard splitting of the given 3-manifold and decide in polynomial time if the manifold contains a nonseparating, 2-sided, closed, incompressible surface.

\section{Preliminaries}

For standard 3-manifold terminologies, we refer the reader to Jaco [7]. The curve complex $C\left(\sum\right)$ for a compact, connected, closed, orientable surface $\sum$ is the abstract simplicial complex whose vertices are isotopy classes of essential, simple closed curves in $\sum$ and whose simplices correspond to sets of distinct isotopy classes which are represented by pairwise disjoint circles (simple closed curves) in $\Sigma$. If $C\left(\sum\right)$ is connected we can define an integer valued distance $d(x, y)$ between a pair of vertices $x, y$ as the number of edges in the shortest edge path between them. Given a handlebody $V$ and a homeomorphism $f: \sum \rightarrow \partial V$, the handlebody set (or a disk complex) $\mathbb{H}$ is the set of (isotopy classes of) circles in $\sum$ that bound properly embedded essential discs in $V$. The genus $\mathrm{g}$ boundary set $\mathbb{H}^{g}$ is the set of non-separating circles in $\sum$ such that each bounds a properly embedded, 2-sided, incompressible, genus $g$ surface in $V$ and the boundary set is the union $\mathbb{H}^{\infty}=\cup_{g \geq 0} \mathbb{H}^{g}$. The Hempel distance, denoted by $d\left(V, V^{\prime}\right)$, of the Heegaard splitting $\left(V, V^{\prime} ; \quad \Sigma_{g}\right)$ as defined in Hempel [8] is given by $d\left(V, V^{\prime}\right)$ $=\inf \left\{d(x, y): x\right.$ is a vertex of $\mathbb{H}, y$ is a vertex of $\left.\mathbb{H}^{\prime}\right\}$.

The boundary distance as defined in Johnson and Patel [6] is given by

$$
d^{\infty}(\Sigma)=d\left(\mathbb{H}^{\infty}, \mathbb{H}^{\prime \infty}\right) .
$$

\section{Homology Curve Complex}

Let $\Sigma_{g}$ denote a closed, connected and oriented surface of genus $g>1$. It is well-known that $H_{1}\left(\Sigma_{g} ; \mathbb{Z}\right)$ has the standard bilinear intersection form $\langle\cdot, \cdot\rangle$, which is symplectic (i.e., $\langle x, x\rangle=0, \forall x \in H_{1}\left(\Sigma_{g} ; \mathbb{Z}\right)$ and the form establishes a self-duality on $H_{1}\left(\Sigma_{g} ; \mathbb{Z}\right)$ (i.e. an isomorphism $\left.H_{1}\left(\Sigma_{g} ; \mathbb{Z}\right) \rightarrow \operatorname{Hom}_{\mathbb{Z}} H_{1}\left(\Sigma_{g} ; \mathbb{Z}\right), \mathbb{Z}\right)$ ), see for example Johnson [9]. For $x, y \in H_{1}\left(\sum_{g} ; \mathbb{Z}\right)$, the integer $\langle x, y\rangle$ is called the algebraic intersection number between $x$ and $y$. A basis of $H_{1}\left(\sum_{g} ; \mathbb{Z}\right)$ ) (which is a free $\mathbb{Z}$ module of rank $2 g$ ) is called canonical or symplectic if it is of the form $\left\{a_{1}, \cdots, a_{g}, b_{1}, \cdots, b_{g}\right\}$ with $\left\langle a_{i}, a_{j}\right\rangle=\left\langle b_{i}, b_{j}\right\rangle$ $=0$, and $\left\langle a_{i}, b_{j}\right\rangle=\delta_{i j} \quad$ (Kronecker delta), $i, j \in\{1,2, \cdots$, $g\}$. It is well-known that $H_{1}\left(\Sigma_{g} ; \mathbb{Z}\right)$ has the standard canonical basis (see Figure 1).

A nontrivial element $x \in H_{1}\left(\sum_{g} ; \mathbb{Z}\right)$ is said to be primitive (relative to the canonical basis) if its coordinates are relatively prime.

\subsection{Definition}

We define the homology curve complex of the surface $\Sigma_{g}$, denoted by $H C\left(\Sigma_{g}\right)$, to be an abstract simplicial complex whose vertices are the primitive elements of $H_{1}\left(\sum_{g} ; \mathbb{Z}\right)$ and a collection $\left\{c_{1}, \cdots, c_{m}\right\}$ of $\mathrm{m}$ distinct vertices form an $(m-1)$-simplex if the algebraic intersection numbers $\left\langle c_{i}, c_{j}\right\rangle=0, \forall i, j \forall\{1, \cdots, m\}$. In the following, we shall represent a vertex of $H C\left(\Sigma_{g}\right)$ by its coordinates relative to the standard canonical (ordered) basis B.

\subsection{Lemma}

For $g>1$, if $x, y$ are distinct vertices of $H C\left(\Sigma_{g}\right)$, then there is a vertex $z$ of $H C\left(\Sigma_{g}\right)$ such that $\langle x, z\rangle=0=\langle y, z\rangle$.

Proof. The standard intersection form $\langle\cdot, \cdot\rangle$ on $H_{1}\left(\sum_{g} ; \mathbb{Z}\right)$ induces a module homomorphism $f: H_{1}\left(\sum_{g} ; \mathbb{Z}\right) \rightarrow \mathbb{Z} \quad \times$ $\mathbb{Z}$ defined by $t \mapsto(\langle t, x\rangle,\langle t, y\rangle)$. Since $\mathbb{Z}$ is a PID, $\operatorname{ker}(f)$ and $\operatorname{im}(f)$ are free submodules of the free $\mathbb{Z}$-modules $H_{1}\left(\Sigma_{g} ; \mathbb{Z}\right)$ and $\mathbb{Z} \times \mathbb{Z}$ respectively and the sequence $0 \rightarrow \operatorname{ker}(f) \rightarrow H_{1}\left(\Sigma_{g} ; \mathbb{Z}\right) \rightarrow \operatorname{im}(f) \rightarrow 0$ is split-exact. So, $H_{1}\left(\Sigma_{g} ; \mathbb{Z}\right)=\operatorname{ker}(f) \oplus \operatorname{im}(f)$. Since $\operatorname{dim}_{\mathbb{Z}}(\operatorname{ker}(f))=$ $\operatorname{dim}_{\mathbb{Z}} H_{1}\left(\sum_{g} ; \mathbb{Z}\right)-\operatorname{dim}_{\mathbb{Z}}(\operatorname{im}(f)) \geq 2 g-2 \geq 2$, we get a nontrivial element $z \in H_{1}\left(\Sigma_{g} ; \mathbb{Z}\right)$ satisfying $\langle x, z\rangle=0=$ $\langle y, z\rangle$ and so by dividing each of the co-ordinates of $z$ by their $g c d$, if necessary, we can always take $z$ to be a primitive element.

\subsection{Lemma}

For $g>1, H C\left(\sum_{g}\right)$ is an infinite dimensional, connected abstract simplicial complex and if the integer valued distance $d_{H}(x, y)$ between a pair of vertices $x, y$ is the number of edges in the shortest edge path between them, then $d_{H}$ is explicitly given by

$$
d_{H}(x, y)=\left\{\begin{array}{l}
0, \text { if } x=y \\
1, \text { if } x \neq y \text { and } x, y=0 ; \\
2, \text { otherwise. }
\end{array}\right.
$$

Proof. It is easy to see that $\forall m \in \mathbb{N}$, the set $A_{m}=\{(1$, $\left.x, 0, \cdots, 0) \in \mathbb{Z}^{2 \mathrm{~g}}: 1 \leq x \leq m, x \in \mathbb{N}\right\}$ of vertices of $H C\left(\Sigma_{g}\right)$ forms an $(m-1)$-simplex in $H C\left(\Sigma_{g}\right)$. There-

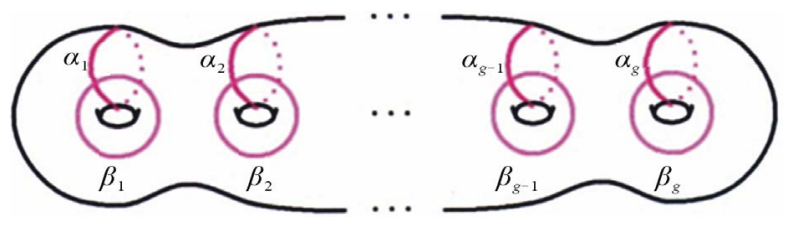

Figure 1. A representative of a canonical basis for $H_{1}\left(\Sigma_{g} ; \mathbb{Z}\right)$. 
$H C\left(\sum_{g}\right)$ is infinite dimensional. To see that $H C\left(\sum_{g}\right)$ is connected, let $x, y$ be distinct vertices of $H C\left(\sum_{g}\right)$.

If $\langle x, y\rangle=0$, then they can be joined by an edge by definition; otherwise by lemma (3.2), there is a vertex $z$ such that $x$ can be joined to $z$ by an edge and then $z$ can be joined to $y$ by another edge.

Notice that a circle $\alpha$ on $\sum_{g}$ is separating if and only if its homology class is zero. There is a characterization of the vertex set of $H C\left(\sum_{g}\right)$.

\subsection{Lemma}

(Meyerson [10]) A nontrivial element of $H_{1}\left(\Sigma_{g} ; \mathbb{Z}\right)$ can be represented by a non-separating circle on $\sum_{g}$ if and only if it is primitive relative to the standard canonical (ordered) basis of $H_{1}\left(\Sigma_{g} ; \mathbb{Z}\right)$.

\subsection{Theorem}

For $g>1$, there is an explicit algorithm which takes as input $\sum_{g}$, a canonical ordered basis $B=\left\{a_{1}, \cdots, a_{g}\right.$, $\left.b_{1}, \cdots, b_{g}\right\}$ for the homology $H_{1}\left(\sum_{g} ; \mathbb{Z}\right)$, a pair of vertices $\alpha, \beta$ of $H C\left(\sum_{g}\right)$ and returns the distance between them.

Proof. By part $(f)$ of Theorem 1 in [11], there is an algorithm to compute the algebraic intersection number $\langle\alpha, \beta\rangle$ and also the co-ordinates of $\alpha$ and $\beta$ relative to the basis $\mathrm{B}$ in polynomial time. If $\alpha=\beta$ then by lemma 3.3 $d_{H}(\alpha, \beta)=0$. If $\alpha \neq \beta$ by lemma 3.3 again, $d_{H}(\alpha, \beta)=1$ if and only if the algebraic intersection number between $\alpha$ and $\beta=0$. If neither of these hold, then by lemma 3.3 again $d_{H}(\alpha, \beta)=2$.

\subsection{Proposition}

With the same notations as above, every simplicial automorphism $\lambda: H C\left(\sum_{g}\right) \rightarrow H C\left(\sum_{g}\right)$ induces an isometry on the metric space $\left(H C^{0}\left(\Sigma_{g}\right), d_{H}\right)$, where $H C^{0}\left(\Sigma_{g}\right)$ denotes the set of vertices of $H C\left(\sum_{g}\right)$.

Proof. Let $x, y$ be vertices of $H C\left(\sum_{g}\right)$ with $d_{H}(x, y)=$ $k$, where $k=0,1$, or 2 . For $k=0,1$, the result follows from the fact that a simplicial automorphism maps $\mathrm{m}$ simplices to m-simplices and in particular if $x \neq y$ and $\langle x, y\rangle=0$, thereby $d_{H}(x, y)=1$, then $x, y$ form an edge. So, $\lambda(x) \neq \lambda(y)$ will also form an edge, so $\langle\lambda(x), \lambda(y)\rangle=0$ thereby $d_{H}(\lambda(x), \lambda(y))=1$. If $k=2$, i.e. if $\langle x, y\rangle \neq 0$, by lemma (3.2) there is a vertex $\mathrm{z}$ of $H C\left(\sum_{g}\right)$ such that $\langle x, z\rangle=0=\langle y, z\rangle$. So, $\langle\lambda(x), \lambda(z)\rangle=0=\langle\lambda(y), \lambda(z)\rangle$. This gives, by triangle inequality, $d_{H}(\lambda(x), \lambda(y)) \leq 2$. However, $d_{H}(\lambda(x), \lambda(y))<2$ implies that $d_{H}(\lambda(x), \lambda(y))=0$ or 1 . This implies that $\langle\lambda(x), \lambda(y)\rangle=0$ and so $\langle x, y\rangle=0$, which is a contradiction. So, $d_{H}(\lambda(x), \lambda(y))=2$.

\subsection{Proposition}

Let $k \in\{0,1,2\}$. For $g>1$, the set $\left\{(x, y) \in H C^{0}\left(\sum_{g}\right) \times\right.$
$\left.H C^{0}\left(\sum_{g}\right) \mid d_{H}(x, y)=k\right\}$ is infinite.

Proof. For $k=0,1$, the result follows from lemma (3.3). For $k=2$, there are vertices $\mathrm{a}, \mathrm{b}$ in the vertex set of $H C\left(\sum_{g}\right)$ with algebraic intersection number 1 , which also have respective representative circles with geometric intersection number 1 . Consider the positive Dehn twist $T_{a}$. Now, $\forall n \in \mathbb{Z},\left\langle T_{a}^{n}(b), b\right\rangle=\langle b+n\langle a, b\rangle a, b\rangle=$ $n\langle a, b\rangle\langle a, b\rangle+\langle b, b\rangle=n$ and so by definition, $d_{H}\left(T_{a}^{n}(b), b\right)=2$, whenever $n \neq 0$. This proves the result.

\section{Applications}

As an application of the concepts developed in the previous sections we shall present a complexity measure for Heegaard splitting using the homology curve complex and prove.

\subsection{Theorem}

(Theorem 1.1) Given a closed, connected, orientable 3manifold $M$ and a Heegaard splitting $M=V \cup_{\Sigma g} V^{\prime}$ of genus $g>1$, there is an algorithm, which runs in polynomial time, to decide whether $M$ contains a non-separating, 2-sided, closed incompressible surface.

Before giving the proof we need to introduce some more notations and definitions:

\subsection{Definition}

For a Heegaard splitting $\left(V, V^{\prime} ; \sum_{g}\right)$, let $\mathfrak{D}$ (resp. $\left.\mathfrak{D}^{\prime}\right)$ denote the set of vertices in $H C\left(\Sigma_{g}\right)$, each represented by a circle whose homology class is nontrivial in $\Sigma_{g}$, but trivial in $V$ (resp. $\left.V^{\prime}\right)$. Then, we define the $H$-distance, denoted by $d_{H}\left(V, V^{\prime}\right)$, for the splitting $\left(V, V^{\prime} ; \Sigma_{g}\right)$ to be the $\inf \left\{d_{H}(x, y): x \in \mathfrak{D}, y \in \mathfrak{D}^{\prime}\right\}$.

As immediate consequence of lemma (3.3), we have the following.

\subsection{Lemma}

For $(g>1)$, the H-distance of the Heegaard splitting $M=V \cup_{\Sigma g} V^{\prime}$ takes only the finite values 0,1 or 2 .

\subsection{Remark}

For any Heegaard splitting $M=V \cup_{\Sigma g} V^{\prime}$ of genus 3 or greater, Johnson and Patel [6] showed, using complex of curves (different from homology curve complex), an analogous result that distance $d^{\infty}\left(\sum_{g}\right)$ is equal to 0,1 or 2.

\subsection{Definition}

A complete meridian system (or simply $\mathrm{cms}$ ) for a handlebody $V$ is a set $L=\left\{D_{1}, \cdots, D_{g}\right\}$ of pairwise disjoint properly embedded discs on $\mathrm{V}$ such that the manifold obtained by cutting $V$ open along $L$ is a 3-ball. The 
boundaries of the discs of a cms for $V$ form a meridian system for the surface $\partial V$.

Given a cms $L=\left\{D_{1}, \cdots, D_{g}\right\}$ for a handlebody $V$, let $\alpha$ be a simple arc on the boundary $\partial V$ connecting $\partial D_{i}$ and $\partial D_{j}(i \neq j)$ with int $(\alpha)$ disjoint from $L$. Let $N$ denote a regular neighbourhood of $\partial D_{i} \cup \partial D_{j} \cup \alpha$ in $\Sigma_{g}$. Then, $\partial N$ has three components, one is a copy of $\partial D_{i}$, one is a copy of $\partial D_{j}$ and the other one is called a band sum of $\partial D_{i}$ and $\partial D_{j}$ along $\alpha$ on $\partial V$ which gives arise to a compressing disc $D_{i j}$ in $V$ (after slight pushing the interior of the disc inside $V$ ) with boundary $\partial D_{i j}$ being the band sum. Denote $L^{\prime}=\left\{D_{1}, \cdots, D_{i-1}, D_{i j}, D_{i+1}, \cdots, D_{g}\right\}$. We say that $L^{\prime}$ is obtained from L by an elementary slide (and $D_{i j}$ is also called a disc slide). If a cms $L^{\prime}$ on $V$ is obtained from a cms $L$ on $V$ via a finite number of elementary slides and isotopies, we say that $L$ and $L^{\prime}$ are equivalent and denoted by $L \sim L^{\prime}$. We also say that $L$ slides to $L^{\prime}$. The following fact about cmss of discs on a handlebody $V$ is wellknown (see for example [12]), which tells us how two cmss of discs for a handlebdy are related.

\subsection{Proposition}

Assume that $L$ is a cms of discs for a handlebody $V$ and $L$ slides to $L^{\prime}$, then $L^{\prime}$ is also a cms of discs for $V$. Conversely, any two cmss of discs for $V$ are equivalent.

\subsection{Remark}

It is well-known that if $D$ is a non-separating properly embedded compressing disc for a genus g handlebody $V$, then $\{D\}$ can be extended algorithmically to a $\mathrm{cms}$ of discs for $V$ in $g$ many steps.

Proof of the theorem (4.1). We will begin with the following characterization of a closed connected orientable 3-manifold which is an extension of Johnson-Patel ([6], lemma 4) to the setting of homology curve complex.

\subsection{Theorem}

Let $M$ be a closed connected orientable 3-manifold with a Heegaard splitting $\left(V, V^{\prime} ; \sum_{g}\right)$ of genus $g>1$. Then for any pair of cmss $L=\left\{D_{1}, \cdots, D_{g}\right\}, L^{\prime}=\left\{D_{1}^{\prime}, \cdots, D_{g}^{\prime}\right\}$ for the respective handlebodies $V$ and $V^{\prime}$, the following statements are equivalent:

1) $M$ contains a non-separating, two-sided, closed incompressible surface;

2) $H_{1}(M)$ is infinite;

3) The matrix $A=\left(a_{i j}\right)$, where $a_{i j}$ is the algebraic intersection number of $D_{i}$ and $\partial D_{j}^{\prime}$, is singular;

4) $d^{\infty}\left(\sum_{g}\right)=0$

5) $d_{H}\left(V, V^{\prime}\right)=0$.

Proof. $1 \Rightarrow 2$ is a classical result (see Jaco [7], or Johnson-Patel [6]). $2 \Rightarrow 3 \Rightarrow 4$ is due to JohnsonPatel ([6], lemma 4). By definition $\mathfrak{D}$ is a quotient of
$\mathbb{H}^{\infty}$, consisting of homology classes of elements of $\mathbb{H}^{\infty}$, so $d^{\infty}\left(\sum_{g}\right)=0$ implies $d_{H}\left(V, V^{\prime}\right)=0$, or $4 \Rightarrow 5$. Now we prove that $5 \Rightarrow 1$. If $d_{H}\left(V, V^{\prime}\right)=0$ then there exists $x$ $\in \mathfrak{D} \cap \mathfrak{D}^{\prime} \subset H C\left(\sum_{g}\right)$. Pick two disjoint simple closed curves $\ell$ and $\ell^{\prime}$ on $\sum_{g}$, each of which represents $x$. Then, $\ell$ and $\ell^{\prime}$ bound two-sided, non-separating properly embedded compact surfaces $F \subset V$ and $F^{\prime} \subset V^{\prime}$ respectively. Since $\ell$ and $\ell^{\prime}$ are homologous in $\sum_{g}$, they cobound a compact subsurface $S$ of $\Sigma_{g}$. The union $F \cup S \cup F^{\prime}$ is a two-sided, non-separating closed surface embedded in $M$. By compressing repeatedly, if necessary, we eventually find a closed, non-separating, two-sided incompressible surface in $M$.

We are ready to complete the proof of theorem (4.1).

Proof. (Completion of proof of theorem (4.1))

By theorem (4.8), existence of a non-separating, twosided, closed incompressible surface in $\mathrm{M}$ is reduced to examining if $d_{H}\left(V, V^{\prime}\right)=d_{H}\left(\mathfrak{D}, \mathfrak{D}^{\prime}\right)=d^{\infty}\left(\Sigma_{g}\right)=0$, which in turn reduced to examining if the matrix $A=\left(a_{i j}\right)$ is singular, where $a_{i j}$ is the algebraic intersection number of $D_{i}$ and $\partial D_{j}$. One can compute the entries $a_{i j}$ of the matrix $A$ in polynomial time (see for example [11] theorem 1 (f)) and the non-singularity of a matrix can be determined in polynomial time (see for example section 2.3 of [13]). This completes the proof of the theorem (4.1).

\subsection{Problem}

It will be interesting to characterize those closed orientable 3-manifolds which possess Heegaard splittings having H-distance 1 and/or 2.

\section{Acknowledgements}

We acknowledge Prof. Joan Birman for her useful comments on an earlier version of the draft of our paper. We also acknowledge the anonymous referees for their useful comments.

\section{REFERENCES}

[1] W. Jaco and U. Oertel, "An Algorithm to Decide If a 3Manifold Is a Haken Manifold," Topology, Vol. 23, No. 2, 1984, pp. 195-209. doi:10.1016/0040-9383(84)90039-9

[2] W. Haken, "Theorie der Normalflachen," Acta Mathematica, Vol. 105, No. 3-4, 1961, pp. 245-375. doi:10.1007/BF02559591

[3] W. Jaco, D. Letscher and J. H. Rubinstein, "Algorithms for Essential Surfaces in 3-Manifolds," Contemporary Mathematics, Vol. 314, 2002, pp. 107-124. doi: $10.1090 /$ conm $/ 314 / 05426$

[4] J. Birman, "The Topology of 3-Manifolds, Heegaard Distances and the Mapping Class Group of a 2-Manifold," arXiv.org, 2005. http://arxiv.org/abs/math/0502545 
[5] I. Irmer, "Geometry of the Homology Curve Complex," arXiv.org, 2011. http://arxiv.org/abs/1107.3547

[6] J. Johnson and T. Patel, "Generalized Handlebody Sets and Non-Haken 3-Manifolds," Pacific Journal of Mathematics, Vol. 235, No. 1, 2005, pp. 35-41.

[7] W. Jaco, "Lectures on Three-Manifold Topology," CBMS Regional Conference Series in Mathematics, American Mathematical Society, Providence, 1980.

[8] J. Hempel, "3-Manifolds as Viewed from the Curve Complex," Topology, Vol. 40, No. 3, 2001, pp. 631-657. doi:10.1016/S0040-9383(00)00033-1

[9] D. Johnson, "An Abelian Quotient of the Mapping Class Group $\mathrm{I}_{g}$," Mathematische Annalen, Vol. 249, No. 3, 1980, pp. 225-242. doi:10.1007/BF01363897

[10] M. D. Meyerson, "Representing Homology Classes of
Closed Orientable Surfaces," Proceedings of AMS, Vol. 61, No. 1, 1976, pp. 181-182.

[11] M. Schaefer, E. Sedgwick and D. Stefankovic, "Algorithms for Normal Curves and Surfaces," Lecture Notes in Computer Science, Springer, 2002, New York, pp. 370380.

[12] F. C. Lei, "Complete Systems of Surfaces in 3-Manifolds," Mathematical Proceedings of the Cambridge Philosophical Society, Vol. 122, No. 1, 1997, pp. 185-191. doi:10.1017/S0305004196001545

[13] D. Saunders and Z. D. Wan, "Smith Normal Form of Dense Integer Matrices, Fast Algorithms into Practice," Proceedings of the 2004 International Symposium on Symbolic and Algebraic Computation, 4-7 July 2004, Santander. 